\title{
The $\gamma$ TuRC Revisited: A Comparative Analysis of Interphase and Mitotic Human $\gamma$ TuRC Redefines the Set of Core Components and Identifies the Novel Subunit GCP8
}

\author{
Neus Teixidó-Travesa, ${ }^{*+}$ Judit Villén, $^{\ddagger}$ Cristina Lacasa, ${ }^{*}$ Maria Teresa Bertran, ${ }^{+}$ \\ Marco Archinti, ${ }^{*}$ Steven P. Gygi, ${ }^{\ddagger}$ Carme Caelles, ${ }^{+\$}$ Joan Roig, $^{+}$and Jens Lüders*
}

${ }^{*}$ Cell and Developmental Biology Programme and ${ }^{+}$Molecular Medicine Programme, Institute for Research in Biomedicine (IRB), and \$Department of Biochemistry and Molecular Biology (Pharmacy), Universitat de Barcelona, 08028 Barcelona, Spain; and łDepartment of Cell Biology, Harvard Medical School, Boston, MA 02115

Submitted May 10, 2010; Revised July 27, 2010; Accepted September 13, 2010

Monitoring Editor: Monica Bettencourt-Dias

The $\gamma$-tubulin complex is a multi-subunit protein complex that nucleates microtubule polymerization. $\gamma$-Tubulin complexes are present in all eukaryotes, but size and subunit composition vary. In Drosophila, Xenopus, and humans large $\gamma$-tubulin ring complexes ( $\gamma$ TuRCs) have been described, which have a characteristic open ring-shaped structure and are composed of a similar set of subunits, named $\gamma$-tubulin, GCPs 2-6, and GCP-WD in humans. Despite the identification of these proteins, $\gamma \mathrm{TuRC}$ function and regulation remain poorly understood. Here we establish a new method for the purification of native human $\gamma$ TuRC. Using mass spectrometry of whole protein mixtures we compared the composition of $\gamma$ TuRCs from nonsynchronized and mitotic human cells. Based on our analysis we can define core subunits as well as more transient interactors such as the augmin complex, which associates specifically with mitotic $\gamma$ TuRCs. We also identified GCP8/MOZART2 as a novel core subunit that is present in both interphase and mitotic $\gamma$ TuRCs. GCP8 depletion does not affect $\gamma$ TuRC assembly but interferes with $\gamma$ TuRC recruitment and microtubule nucleation at interphase centrosomes without disrupting general centrosome structure. GCP8-depleted cells do not display any obvious mitotic defects, suggesting that GCP8 specifically affects the organization of the interphase microtubule network.

\section{INTRODUCTION}

The $\gamma$-tubulin ring complex $(\gamma \mathrm{TuRC})$ nucleates microtubule polymerization and is a key component of microtubule organizing centers (MTOCs) such as the centrosome. $\gamma$ TuRCs are composed of $\gamma$-tubulin and additional subunits named gamma-tubulin complex proteins (GCPs) in humans (Murphy et al., 1998; Fava et al., 1999; Murphy et al., 2001). Assembly of the $\gamma \mathrm{TuRC}$ involves subcomplexes composed of two molecules of $\gamma$-tubulin and one of each GCP2 and GCP3 ( $\gamma$-tubulin small complex, $\gamma$ TuSC) (Oegema et al., 1999; Kollman et al., 2008). According to a current model the additional GCPs 4-6 promote assembly of $\sim 13 \gamma \mathrm{TuSC}$ into the higher order ring-shaped $\gamma \mathrm{TuRC}$ by forming a stabilizing cap on one side of the ring (Moritz et al., 2000). However,

This article was published online ahead of print in $M B O C$ in Press (http://www.molbiolcell.org/cgi/doi/10.1091/mbc.E10-05-0408) on September 22, 2010.

Address correspondence to: Jens Lüders (jens.luders@irbbarcelona. org) or Joan Roig (joan.roig@irbbarcelona.org).

Abbreviations used: PCM, Pericentriolar material; $\gamma \mathrm{TuRC}, \gamma$-tubulin ring complex.

(C) 2010 N. Teixidó-Travesa et al. This article is distributed by The American Society for Cell Biology under license from the author(s). Two months after publication it is available to the public under an Attribution-Noncommercial-Share Alike 3.0 Unported Creative Commons License (http://creativecommons.org/licenses/by-nc-sa/3.0). in Drosophila only $\gamma$-tubulin and the orthologues of GCP2 and 3 are essential and in the absence of the GCP4- 6 orthologues $\gamma$-tubulin is still recruited to centrosomes and supports microtubule nucleation (Verollet et al., 2006). Similar results were recently obtained in the fungus Aspergillus (Xiong and Oakley, 2009). It is likely that in these cases as well as in budding yeast, which naturally lacks orthologues of GCP4-6, assembly of the $\gamma$ TuSC into a higher order ring structure might only occur upon interaction with MTOCs. In addition to promoting assembly of cytoplasmic $\gamma \mathrm{TuRCs}$, GCPs 4-6 might have other regulatory functions, for example in controlling microtubule length and dynamics, either directly or by interaction with other proteins (Fujita et al., 2002; Venkatram et al., 2004; Bouissou et al., 2009). Orthologues of the $\gamma \mathrm{TuRC}$ subunits GCP2-6 can be found in all eukaryotes containing the $\gamma \mathrm{TuRC}$, suggesting that the GCPs are critical for $\gamma \mathrm{TuRC}$ structure and function. However, as the requirements for microtubule nucleation and organization vary dramatically between cells from such different organisms as yeast and humans, it is likely that with increasing cellular complexity the number of $\gamma \mathrm{TuRC}$ subunits or interactors that participate in $\gamma \mathrm{TuRC}$ function also increases. An example is the GCP-WD subunit (also known as NEDD1), which plays an essential role in the assembly of mitotic and meiotic spindles in animals and plants, but is not found in yeast or Aspergillus (Luders et al., 2006; Haren et al., 2006; Zeng et al., 2009; Ma et al., 2010). GCP-WD has no sequence similarity with the other GCPs and is dispensable for $\gamma \mathrm{TuRC}$ assembly. Instead, GCP-WD functions as a tar- 
geting factor for the $\gamma \mathrm{TuRC}$, mediating its interaction with centrosomes and, in mitosis, also with the mitotic spindle (Luders et al., 2006; Haren et al., 2006). Spindle targeting of the $\gamma \mathrm{TuRC}$ involves mitotic phosphorylation of GCP-WD, which promotes interaction with the human augmin complex (also known as HAUS complex) (Luders et al., 2006; Goshima et al., 2008; Zhu et al., 2008; Lawo et al., 2009; Uehara et al., 2009). According to the so-called amplification model augmin and $\gamma \mathrm{TuRC}$ are laterally bound to spindle microtubules and cooperate in the generation of additional microtubules within the forming spindle, possibly by yTuRC-mediated nucleation (Luders and Stearns, 2007; Goshima et al., 2008). During mitosis GCP-WD also plays a role in the chromatin-dependent nucleation pathway, but the molecular details are not known (Luders et al., 2006).

Our knowledge about the composition of $\gamma \mathrm{TuRCs}$ is derived from the analysis of $\gamma \mathrm{TuRC}$ from various sources such as Drosophila embryos, Xenopus oocytes, and cultured human cells (Zheng et al., 1995; Moritz et al., 1998; Murphy et al., 1998; Fava et al., 1999; Oegema et al., 1999; Murphy et al., 2001). In biochemical terms, the $\gamma \mathrm{TuRC}$ prepared from early Drosophila embryos and CSF-arrested Xenopus oocytes, respectively, most likely resemble the mitotic state, whereas the human $\gamma \mathrm{TuRC}$ isolated from asynchronously growing cultured cells can be considered a nonmitotic, interphase form. A more recent large-scale analysis of mitotic protein complexes has identified additional interactors of the $\gamma \mathrm{TuRC}$ in mitosis (Hutchins et al., 2010). However, whether the $\gamma \mathrm{TuRC}$ undergoes cell cycle-dependent changes in subunit composition or in its interaction with other proteins has not been investigated.

Here we have performed a comparative analysis of the composition of human $\gamma \mathrm{TuRC}$ from asynchronous and mitotic cells by mass spectrometry. Our analysis reveals an interaction network comprising constitutive as well as more transient interactions and identifies a novel $\gamma \mathrm{TuRC}$ core subunit.

\section{MATERIALS AND METHODS}

\section{Molecular Biology}

Full-length Fam128B was PCR amplified from a human liver cDNA library using the following primers: CCGCTCGAGCGATGGCGGCGCAGGGCGTAGG and CCGGAATTCCTAGGTGCTGCCCTGCGTAGGGCT. Full-length Fam128A was PCR-amplified from plasmid pCMV-SPORT6-Fam128A (clone \# 3862861; Open Biosystems, Huntsville, AL) using the same primers.

For expression in human cells amplified GCP8 sequences were inserted into pEGFP-C1 (Clontech, Palo Alto, CA) using XhoI and EcoRI restriction sites For the expression in Escherichia coli, the GCP8B sequence was inserted into the pGEX-4T-1 vector (GE Healthcare, Piscataway, NJ) using EcoRI and Xhol restriction sites. A plasmid expressing $\gamma$-tubulin-Myc-TAP was constructed by replacing the His-tag of $\gamma$-tubulin-Myc-His (pTS556) (Murphy et al., 1998) with the PCR-amplified TAP-tag from plasmid pBS1479 (Rigaut et al., 1999).

For RNAi-mediated depletion of GCP8A and GCP8B we designed RNA oligonucleotides targeting the following sequences within the GCP8A/B open reading frames $(100 \%$ conserved between the two): CGACGUGUUCAAGAUCCUG (oligo 1, Silencer Select Pre-designed siRNA, \# s198094, Ambion, Applied Biosystems, Carlsbad, CA) and CUCGCCGUCUUCCAGAUGCUCAAGU (oligo 2, Stealth version, Invitrogen, Carlsbad, CA). Sequence analysis and alignments were performed with Geneious software (Biomatters, Auckland, NewZealand).

RNAi-mediated depletion of GCP-WD was done as previously described (Luders et al., 2006).

\section{Antibodies and Reagents}

To generate anti-GCP8 antibodies, full-length GCP8B was expressed and affinity-purified as a soluble GST fusion protein in E. coli using glutathionesepharose (GE Healthcare), according to the manufacturer's standard protocol. The protein was then used for immunization of rabbits (Antibody Production Service, Facultat de Farmàcia, Universitat de Barcelona, Spain). After passing the rabbit serum over a resin bearing immobilized GST to eliminate GST-reactive antibodies, GCP8-specific antibodies were affinity-purified using GST-GCP8 immobilized on Affi-Gel10.
The following additional antibodies were used in this study: mouse anti$\gamma$-tubulin (GTU-88; Sigma, St. Louis, MO), mouse anti- $\gamma$-tubulin (Exbio, Prague, Czech Republic), rabbit anti- $\gamma$-tubulin (Sigma), mouse anti- $\alpha$-tubulin (DM1A, Sigma), mouse anti-GCP-WD (7D10, Abnova, Walnut, CA), rabbit anti-GCP-WD (Luders et al., 2006), rabbit anti-GCP5 (H-300, Santa Cruz Biotechnology, Santa Cruz, CA), rabbit anti-pericentrin (Luders et al., 2006), mouse anti-GFP (3E6, Invitrogen), rabbit anti-GFP (Torrey Pines Biolabs, Houston, TX), rabbit anti-Cep215 (IHC-00063, Bethyl Laboratories, Montgomery, TX), rabbit anti-centrin (Groen et al., 2004), rabbit anti-Nek9 (Roig et al., 2002).

Alexa 488- and Alexa 568-conjugated secondary antibodies used for immunofluorescence microscopy were from Invitrogen (Carlsbad, CA), and peroxidase-coupled secondary antibodies for western blotting were from Jackson Immunoresearch Laboratories (West Grove, PA).

\section{Cell Culture, Transfection, and Drug Treatments}

U2OS cell lines were grown in DMEM containing 10\% fetal calf serum.

Cells were transfected with plasmid or siRNA using Lipofectamine 2000 or Lipofectamine RNAiMAX (Invitrogen), respectively.

To generate a Hela S3 cell line stably expressing $\gamma$-tubulin-Myc-TAP cells were transfected with the $\gamma$-tubulin-Myc-TAP expression plasmid and selected in the presence of $0.4 \mu \mathrm{g} / \mathrm{ml}$ geneticin. Resistant clones were isolated and tested for expression of the tagged protein by Western blotting

For interphase microtubule regrowth experiments, dishes containing coverslips with U2OS cells were incubated for $30 \mathrm{~min}$ in an ice-water bath to depolymerize microtubules. To allow microtubule regrowth, coverslips were then incubated in medium at $37^{\circ} \mathrm{C}$, followed by methanol or $4 \%$ paraformaldehyde fixation. For mitotic microtubule regrowth experiments, cells were incubated with $250 \mathrm{ng} / \mathrm{ml}$ nocodazole for $\sim 16 \mathrm{~h}$. Nocodazole was then washed out and microtubules were depolymerized as described above.

\section{Cell Cycle Analysis}

U2OS cells were transfected with GCP8 siRNA for $72 \mathrm{~h}$ and then prepared for flow cytometry by fixation in $70 \%$ ethanol.

Phosphorylated histone $\mathrm{H} 3$ was detected with a phosphospecific polyclonal primary antibody (Millipore, Billerica, MA) and a FITC-conjugated anti-rabbit secondary antibody (Jackson Laboratories). To measure DNA content, cells were stained with propidium iodide (Sigma). Fluorescence was measured by flow cytometry using a Coulter XL Flow Cytometer (Flow Cytometer Unit, Scientific and Technical Services Universitat de Barcelona).

\section{Tandem-Affinity Purification of the Human $\gamma T u R C$}

HeLa S3 $\gamma$-tubulin-Myc-TAP cells were left untreated (for asynchronous samples) or incubated with $250 \mathrm{ng} / \mathrm{ml}$ nocodazole for $\sim 16 \mathrm{~h}$ (for mitotic samples). Cells were harvested by trypsinization or, in the case of mitotic cells, by shake-off, washed in PBS, and lysed in lysis buffer (50 mM HEPES, $\mathrm{pH} 7.5,150 \mathrm{mM} \mathrm{NaCl}, 1 \mathrm{mM} \mathrm{MgCl}, 1 \mathrm{mM}$ EGTA, $0.05 \%$ Triton-X100) containing Complete protease and Phostop phosphatase inhibitors (Roche, Indianapolis, IN) for $10 \mathrm{~min}$ on ice and sonicated with 10 -s bursts at maximum power. After centrifugation for $15 \mathrm{~min}$ at $14,000 \mathrm{~g}$ at $4^{\circ} \mathrm{C}$ the $\gamma \mathrm{TuRC}$ was precipitated from the cleared lysates by addition of an equal volume of $9 \%$ polyethylene glycol and incubation for $20 \mathrm{~min}$ on ice (Murphy et al., 2001). After centrifugation for $15 \mathrm{~min}$ at $12,000 \mathrm{~g}$ at $4^{\circ} \mathrm{C}$ the pellet was resuspended in lysis buffer and centrifuged as before. The supernatant was then incubated with IgG Sepharose beads (GE Healthcare) for $2 \mathrm{~h}$ at $4^{\circ} \mathrm{C}$. The beads were pelleted and washed with 10 column volumes of lysis buffer and with 2 column volumes of wash buffer (50 mM HEPES, pH 7.5, $150 \mathrm{mM} \mathrm{NaCl}, 1 \mathrm{mM}$ $\mathrm{MgCl}_{2}, 0.05 \%$ Triton-X100) containing phosphatase inhibitors. Beads were resuspended in 2 column volumes of wash buffer, and AcTEV protease (Invitrogen) was added to the mixture. After incubation for $2 \mathrm{~h}$ at $16^{\circ} \mathrm{C}$ beads were pelleted. The supernatant was adjusted to $1 \mathrm{mM}$ imidazole and $2 \mathrm{mM}$ calcium chloride and incubated with Calmodulin Sepharose beads (GE Healthcare) for $1 \mathrm{~h}$ at $4^{\circ} \mathrm{C}$. Then the beads were washed with 10 column volumes of wash buffer containing $1 \mathrm{mM}$ imidazole and $2 \mathrm{mM}$ calcium chloride. Beads were pelleted and purified $\gamma$ TuRC was eluted by addition of 5 column volumes of elution buffer (wash buffer with $2 \mathrm{mM}$ EGTA). For the analysis by SDS-PAGE the $\gamma$ TuRC was concentrated using Vivaspin 6 concentrators (Sartorius, Göttingen, Germany).

\section{Mass Spectrometry}

Analysis of individual protein bands: purified $\gamma$ TuRC was separated by SDS-PAGE and stained with Coomassie. Bands were excised from the gel and analyzed by the Proteomics Platform of the Barcelona Science Park, University of Barcelona. Briefly, excised bands were washed sequentially with 25 $\mathrm{mM} \mathrm{NH} \mathrm{HCO}_{3}$ and acetonitrile. Proteins were reduced and alkylated by treatment with $10 \mathrm{mM}$ DTT for $15 \mathrm{~min}$ at $56^{\circ} \mathrm{C}$ and $55 \mathrm{mM}$ iodine acetamide for $15 \mathrm{~min}$, respectively. After sequential washings with buffer and acetronitrile, proteins were digested overnight at $37^{\circ} \mathrm{C}$ with $80 \mathrm{ng}$ of trypsin. Tryptic peptides were extracted from the gel matrix with $10 \%$ formic acid and acetonitrile. Mass spectrometry analysis was done by liquid-chromatography coupled to a Q-TOF mass spectrometer (capLC-ESI-Q-TOF, Micromass- 
Waters). The peptide digests were redissolved in $25 \mu \mathrm{l}$ of $1 \%$ formic acid solution and injected and separated in a C18 reverse phase column (PepMap column, LC Packings, Sunnyvale, CA). Data were processed in MassLynx 4.1, and $\mathrm{pkl}$ files were generated using ProteinLynx software. The pkl files were submitted to database searching using the online MASCOT search engine (Matrixscience; http://www.matrixscience.com/).

For analysis of whole protein mixtures, purified $\gamma \mathrm{TuRC}$ was precipitated with $20 \%$ trichloroacetic acid, in-solution digested in $5 \mathrm{ng} / \mu \mathrm{L}$ trypsin (in 50 $\mathrm{mM}$ ammonium bicarbonate, $\mathrm{pH} 8.3,10 \%$ acetonitrile), and analyzed by liquid-chromatography coupled to tandem mass spectrometry (LC-MS/MS). Peptides were separated by reverse phase chromatography across a $90-\mathrm{min}$ gradient on a C18 microcapillary column and online analyzed on an LTQOrbitrap hybrid mass spectrometer. For each cycle, one full MS scan acquired at high mass resolution on the Orbitrap mass analyzer was followed by 10 MS/MS spectra on the linear ion trap from the 10 most abundant ions. MS/MS spectra were searched against the IPI human protein sequence database using the Sequest algorithm. Peptide matches were filtered to $<0.1 \%$ false-positives using a target-decoy database strategy (Elias and Gygi, 2007); protein false positives were $<1 \%$. Final lists of proteins interacting with $\gamma$ TuRCs from asynchronous and mitotic cell populations were obtained by subtracting protein matches that were also found in an untagged control sample.

\section{Immunoprecipitation}

For immunoprecipitation of EGFP-tagged GCP8 transfected U2OS cells were washed in PBS and lysed (50 mM HEPES, pH 7.5, $150 \mathrm{mM} \mathrm{NaCl}, 1 \mathrm{mM}$ $\mathrm{MgCl}_{2}, 1 \mathrm{mM}$ EGTA, $0.5 \%$ NP-40, protease inhibitors) for $10 \mathrm{~min}$ on ice. After centrifugation for $15 \mathrm{~min}$ at $16,000 \mathrm{~g}$ at $4^{\circ} \mathrm{C}$ cleared lysates were incubated with anti-GFP antibodies for $1 \mathrm{~h}$ at $4^{\circ} \mathrm{C}$. Sepharose Protein G beads (GE Healthcare) were added and the mixture was incubated for an additional hour at $4^{\circ} \mathrm{C}$. The beads were pelleted and washed three times with lysis buffer. Samples were prepared for SDS-PAGE by boiling in sample buffer.

\section{Sucrose Gradient Centrifugation}

U2OS cell extracts were prepared as described above. Two hundred microliters of extract was then loaded on a $4.2 \mathrm{ml} \mathrm{10-40 \%} \mathrm{sucrose} \mathrm{gradient} \mathrm{prepared}$ in $50 \mathrm{mM}$ HEPES, pH 7.5, $150 \mathrm{mM} \mathrm{NaCl}, 1 \mathrm{mM} \mathrm{MgCl}, 1 \mathrm{mM}$ EGTA and centrifuged in a SW-55Ti rotor (Beckman, Brea, CA) for $4 \mathrm{~h}$ at 55,000 rpm at $4^{\circ} \mathrm{C}$. Fractions were collected and analyzed by Western blotting. Aldolase (158 $\mathrm{K}, 7 \mathrm{~S})$ and thyroglobulin (669 K, 19S) (GE Healthcare) were used as molecular weight standards and analyzed in parallel.

\section{Western Blotting}

Cells were washed in PBS and lysed (50 mM HEPES pH 7.5, $150 \mathrm{mM} \mathrm{NaCl}, 1 \mathrm{mM}$ $\mathrm{MgCl}_{2}, 1 \mathrm{mM}$ EGTA, $0.5 \% \mathrm{NP}-40$, protease inhibitors) on ice. Cleared extracts were prepared by centrifugation and subjected to SDS-PAGE. Proteins were transferred to nitrocellulose membranes and probed with antibodies.

\section{Fluorescence Microscopy}

U2OS cells grown on coverslips were fixed in methanol at $-20^{\circ} \mathrm{C}$ for at least 5 min and processed for immunofluorescence. Alternatively, cells were fixed in PBS containing $4 \%$ paraformaldehyde, $0.05 \%$ glutaraldehyde, and $0.1 \%$ Triton X-100 for $15 \mathrm{~min}$ at RT.

Fixed cells were blocked in PBS-BT $(1 \times$ PBS, $0.1 \%$ Triton X-100, and 3\% BSA) and incubated with antibodies in the same buffer. Images were acquired with an Orca AG camera (Hamamatsu, Bridgewater, NJ) on a Leica DMI6000B microscope equipped with 1.4 NA $63 \times$ and $100 \times$ oil immersion objectives. AF6000 software (Leica, Wetzlar, Germany) was used for image acquisition. For further image processing and quantification of fluorescence intensities ImageJ software was used. Intensities were measured in a circular area (2-4 $\mu \mathrm{m}$ diameter) surrounding centrosomes or centrosomal microtubule asters in images acquired with constant exposure settings. For background-correction the intensity measured in an adjacent circular area of equal dimensions in the cytoplasm was subtracted.

\section{RESULTS}

\section{Characterization of the $\gamma T u R C$ Interactome Identifies Constitutive and Mitosis-Specific Interactions}

We developed a new method for the purification of human $\gamma$ TuRCs based on Hela S3 cells stably expressing $\gamma$-tubulin with a C-terminal mycTAP tag. Using this cell line and a modified tandem-affinity purification protocol we were able to produce purified human $\gamma \mathrm{TuRC}$ from nonsynchronized as well as nocodazole-arrested mitotic cells. To analyze the composition of the complexes we performed mass spectrometric analysis of whole protein mixtures. In addition to the known $\gamma$ TuRC subunits, we identified a large number of proteins, some of which were specifically associated with mitotic $\gamma$ TuRCs (Supplemental Table 1). Validating our approach several of these proteins have been described previously as $\gamma \mathrm{TuRC}$ interactors (Table 1) including subunits of the CCT chaperonin complex, which are involved in tubulin folding (Melki et al., 1993), CDK5RAP2 (also known as Cep215), which plays a role in centrosome targeting of $\gamma$-tubulin (Fong et al., 2008; Haren et al., 2009), the AAA+ ATPase RUVBL1, which, together with RUBVBL2, was identified as $\gamma \mathrm{TuRC}$ interactor important for mitotic microtubule assembly (Ducat et al., 2008), and four proteins recently shown to interact with the $\gamma \mathrm{TuRC}$ in mitosis (Hutchins et al., 2010). Of these, MOZART1 plays a role in mitotic spindle assembly (Hutchins et al., 2010), and the other three, MOZART2, NME7, and LGALS3BP, are still uncharacterized. We now demonstrate that all these proteins are associated with both interphase and mitotic yTuRCs (Table 1). As interactors enriched in the mitotic sample we identified seven of the eight subunits of the recently characterized augmin complex, which cooperates with the $\gamma \mathrm{TuRC}$ in noncentrosomal microtubule formation during spindle assembly (Goshima et al., 2008; Lawo et al., 2009; Uehara et al., 2009), and Polo-like kinase 1 , which might regulate mitotic functions of the $\gamma \mathrm{TuRC}$ (Haren et al., 2009; Zhu et al., 2009) (Table 1). In addition to previously described $\gamma$ TuRC interactors, we identified additional proteins that specifically copurified with $\gamma \mathrm{TuRC}$ and represent potential $\gamma \mathrm{TuRC}$ interactors (Supp. Table 1). However, to eliminate false positives these interactions will have to be confirmed by alternative approaches in future studies.

\section{Interphase and Mitotic $\gamma$ TuRCs Have a Similar Core Subunit Composition}

To discriminate between core components of the $\gamma \mathrm{TuRC}$ and more transient interactors, we analyzed purified $\gamma \mathrm{TuRC}$ by PAGE and Coomassie staining, which revealed the most abundant polypeptides. Interphase and mitotic $\gamma$ TuRCs were very similar with respect to subunit composition and relative stoichiometry of subunits (Figure 1). The only obvious difference was a reduced mobility of the GCP-WD band in mitotic $\gamma$ TuRC, which was reported previously and shown to be caused by mitotic phosphorylation (Haren et al., 2006; Luders et al., 2006). Mass spectrometry analysis of the individual most prominent protein bands confirmed the presence of all known $\gamma$ TuRC subunits: $\gamma$-tubulin, GCPs $2-6$, and GCP-WD (Figure $1)$. Interestingly, we consistently observed an additional band at $\sim 20 \mathrm{kDa}$ which was present in both samples with an estimated stoichiometry similar to the other known subunits. Mass spectrometry identified this band as a mixture of the two closely related, uncharacterized proteins MOZART2A and MOZART2B, which were recently described as novel mitotic $\gamma \mathrm{TuRC}$ interactors (Hutchins et al., 2010). We have named these proteins GCP8A and GCP8B to indicate that they are core subunits of the $\gamma \mathrm{TuRC}$ (see below).

In summary our analysis shows that $\gamma$ TuRCs from nonsynchronized and mitotic cells are very similar in their core subunit composition, but they differ with respect to more transient interactions.

\section{GCP8 Is a Novel Core Subunit of the Human $\gamma$ TuRC}

GCP8A and GCP8B polypeptides were present in all of our $\gamma$ TuRC preparations and this association was independent of the $\gamma \mathrm{TuRC}$ cell cycle state. As GCP8A/B were readily visualized by Coomassie staining with intensities comparable to the other known subunits (Figure 1), we speculated that they might represent novel core subunits of the $\gamma \mathrm{TuRC}$. Public databases contain GCP8-related sequences from various 
Table 1. Composition of interphase and mitotic $\gamma$ TuRCs

\begin{tabular}{|c|c|c|c|c|c|c|}
\hline \multirow[b]{2}{*}{ Protein } & \multicolumn{3}{|c|}{ Interphase } & \multicolumn{3}{|c|}{ Mitotic } \\
\hline & $\begin{array}{l}\text { Control } \\
\text { peptides }\end{array}$ & $\begin{array}{c}\gamma \text { TuRC } \\
\text { peptides }\end{array}$ & $\begin{array}{c}\gamma \text { TuRC } \\
\text { coverage, \% }\end{array}$ & $\begin{array}{l}\text { Control } \\
\text { peptides }\end{array}$ & $\begin{array}{c}\gamma \text { TuRC } \\
\text { peptides }\end{array}$ & $\begin{array}{c}\gamma \text { TuRC } \\
\text { coverage, \% }\end{array}$ \\
\hline$\gamma$-Tubulin & 3 & 42 & 61.4 & 1 & 36 & 55.4 \\
\hline GCP2 & 1 & 61 & 52.8 & 0 & 58 & 54.5 \\
\hline GCP3 & 3 & 63 & 46.2 & 0 & 49 & 43.7 \\
\hline GCP4 & 0 & 27 & 53.2 & 0 & 22 & 43.6 \\
\hline GCP5 & 0 & 53 & 45.3 & 0 & 40 & 37.4 \\
\hline GCP6 & 0 & 61 & 37.8 & 0 & 48 & 34.4 \\
\hline GCP-WD & 1 & 33 & 48.0 & 0 & 21 & 39.5 \\
\hline GCP8A & 0 & $5(2)$ & 49.4 & 0 & $3(1)$ & 32.3 \\
\hline GCP8B & 0 & $4(1)$ & 31.0 & 0 & $3(1)$ & 31.0 \\
\hline MOZART1 & 0 & 2 & 13.4 & 0 & 2 & 52.4 \\
\hline NME7 & 0 & 10 & 31.4 & 0 & 9 & 26.6 \\
\hline LGALS3BP & 1 & 5 & 12.3 & 0 & 4 & 10.6 \\
\hline CDK5RAP2 & 0 & 19 & 14.6 & 0 & 7 & 5.3 \\
\hline RUVBL2 & 4 & 17 & 36.6 & 8 & 11 & 30.3 \\
\hline RUVBL1 & 4 & 15 & 32.9 & 2 & 6 & 17.1 \\
\hline TCP1 & 0 & 20 & 38.1 & 0 & 15 & 30.8 \\
\hline CCT2 & 0 & 33 & 60.3 & 0 & 17 & 41.9 \\
\hline ССТ3 & 1 & 20 & 39.5 & 0 & 9 & 18 \\
\hline CCT4 & 0 & 20 & 30.9 & 0 & 9 & 20.6 \\
\hline ССТ5 & 0 & 22 & 29.9 & 0 & 12 & 21.3 \\
\hline ССТ6А & 0 & 12 & 26.2 & 0 & 6 & 18.3 \\
\hline ССТ7 & 0 & 17 & 40.0 & 0 & 12 & 26.5 \\
\hline ССТ8 & 4 & 26 & 38.9 & 0 & 20 & 36.2 \\
\hline HAUS1 & 0 & 0 & 0 & 0 & 1 & 4.0 \\
\hline HAUS2 & 0 & 0 & 0 & 0 & 1 & 6.0 \\
\hline HAUS3 & 0 & 0 & 0 & 0 & 5 & 10.0 \\
\hline HAUS4 & 0 & 0 & 0 & 0 & 3 & 11.6 \\
\hline HAUS5 & 0 & 1 & 2.5 & 0 & 7 & 13.7 \\
\hline HAUS6 & 0 & 1 & 2.1 & 0 & 4 & 7.2 \\
\hline HAUS8 & 0 & 0 & 0 & 0 & 3 & 10.2 \\
\hline PLK1 & 0 & 1 & 3.2 & 0 & 4 & 9.3 \\
\hline
\end{tabular}

Known $\gamma$ TuRC interactors identified by mass spectrometry of whole protein mixtures are listed with the number of unique peptides identified in control and $\gamma$ TuRC-containing samples, respectively. For GCP8A and GCP8B the peptides that uniquely mapped to only one of the two proteins are indicated in brackets. For each protein identified in the $\gamma$ TuRC samples the percentage of sequence coverage is indicated.

species ranging from mammals to marine invertebrates, but we were unable to find similar sequences in plants, Drosophila, $C$. elegans, and yeast. The highest degree of similarity between GCP8 orthologues is found in an N-terminal region that is predicted to be of mainly helical secondary structure (Figure 2A). Human GCP8A and GCP8B are two highly related proteins with no similarity to GCPs 2-6 or GCP-WD. GCP8A and GCP8B are encoded by two distinct genes, but their amino acid sequence is more than $96 \%$ identical. Interestingly, in other species only a single GCP8 gene is present and the encoded proteins are more similar to human GCP8B (Figure 2A), suggesting that GCP8A is the result of a gene duplication in humans. For the remainder of the text we will simply use the term GCP8 when referring to both GCP8 proteins.

We cloned human GCP8B from a human liver cDNA library to characterize its function in detail. To test the interaction of GCP8B with the $\gamma \mathrm{TuRC}$ we expressed GFPtagged GCP8B in human cells and immunoprecipitated the protein with GFP-specific antibodies. Probing with $\gamma$-tubulin-specific antibodies demonstrated that $\gamma$-tubulin coprecipitated with GFP-GCP8B (Figure 2B). To investigate whether GCP8 has properties of a genuine $\gamma$ TuRC subunit we produced an antibody against GCP8, fractionated human cell extract by sucrose gradient centrifugation and probed with the GCP8 antibody. Due to the high degree of sequence similarity between GCP8A and GCP8B this antibody recog- nizes both forms (Supplemental Figure S1). Indeed GCP8 cofractionated with $\gamma$-tubulin and GCP5 with a major peak at a size expected for the $\gamma \mathrm{TuRC}$ (Figure 2C). Interestingly, some GCP8 also cofractionated with $\gamma$-tubulin but not GCP5 at a smaller size, which could represent a $\gamma$ TuRC subcomplex such as the $\gamma$ TuSC. Costaining of GCP8 and $\gamma$-tubulin in fixed human cells revealed localization of GCP8 to centrosomes throughout the cell cycle and to spindle microtubules in mitosis (Figure 2D). Similar results were obtained for GFP-tagged GCP8 after transient expression and staining of fixed cells with GFP- and $\gamma$-tubulin-specific antibodies (Supplemental Figure S2). Together our data are consistent with the interpretation that GCP8 is a novel $\gamma \mathrm{TuRC}$ subunit.

\section{Centrosome Localization of GCP8 Requires GCP-WD}

Centrosome localization of the $\gamma \mathrm{TuRC}$ requires the GCP-WD subunit, which in human cells functions as a targeting factor for the $\gamma$ TuRC (Haren et al., 2006; Luders et al., 2006). We tested whether centrosomal targeting of GCP8 also depends on the presence of GCP-WD. In agreement with previous reports, RNAi-mediated depletion of GCP-WD strongly interfered with centrosomal recruitment of $\gamma$-tubulin both in interphase and mitosis ( $\sim 70 \%$ reduction, respectively; Figure 3, A and B). Interestingly, centrosomal GCP8 was also reduced to a similar degree in interphase and mitotic GCPWD-depleted cells ( $\sim 75 \%$ reduction, respectively; Figure 3 , 


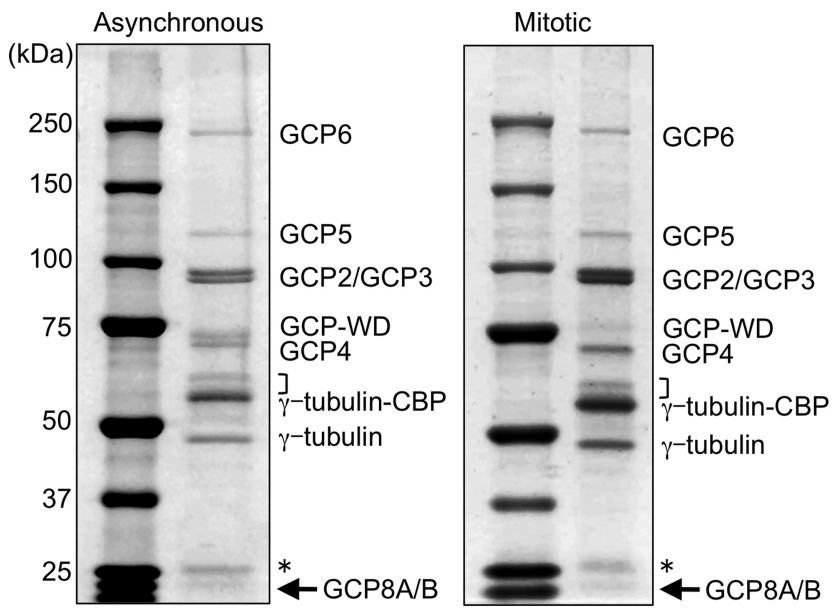

Figure 1. Human $\gamma \mathrm{TuRC}$ s purified from asynchronous and mitotic cells have a similar subunit composition. Hela S3 cells stably expressing TAP-tagged $\gamma$-tubulin were left untreated or arrested in mitosis by nocodazole treatment as indicated. $\gamma \mathrm{TuRC}$ were purified by tandem affinity chromatography and analyzed by SDS-PAGE and Coomassie staining. The position of core subunits as identified by mass spectrometry of excised protein bands are indicated. In the mitotic sample GCP-WD is present in its phosphorylated form and migrates with slightly reduced mobility. The arrow points at the novel subunit GCP8A/B. The asterisk marks contaminating IgG light chain derived from the affinity resin, the bracket indicates the position of CCT subunits.

A and B). Thus, also with respect to its centrosome targeting, GCP8 behaves like a true $\gamma$ TuRC subunit.

\section{GCP8 Is Not Required for the Assembly of Cytoplasmic $\gamma$ TuRCs}

We used RNAi to deplete GCP8 from human cells. All RNAi-derived data were reproduced with two different siRNAs (see Materials and Methods) indicating the specificity of the effects. Western blotting of whole cell lysate $72 \mathrm{~h}$ after transfection indicated that GCP8 was efficiently depleted. Importantly, GCP8 depletion did not significantly affect the total levels of other $\gamma$ TuRC subunits (Figure 4A). We noticed that GCP8-depleted cells had a slightly slower proliferation rate compared with control cells (Figure 4B). Surprisingly, however, FACS analysis indicated that the cell cycle profile after GCP8 depletion was similar to control cells (Supplemental Figure S3). There was no increase in the mitotic index or in the subG1 population of cells, suggesting that the reduced proliferation was not due to mitotic progression defects or cell death (Supplemental Figure S3). To investigate whether the inhibitory effect on cell proliferation might be related to an impaired $\gamma \mathrm{TuRC}$ we tested whether the $\gamma$ TuRC was present in GCP8-depleted cells. We analyzed extracts from control and GCP8-depleted cells by sucrose gradient centrifugation. $\gamma$-Tubulin in control-transfected extracts fractionated mostly at the size of the large $\gamma$ TuRC (Figure 4C) and the profile was comparable to untreated extracts (Figure 2C). GCP8-depleted extracts showed a very similar $\gamma$-tubulin profile indicating that the $\gamma \mathrm{TuRC}$ was not disrupted (Figure 4C). Interestingly, the small amount of GCP8 that was left after the depletion still comigrated with the $\gamma \mathrm{TuRC}$ indicating its tight association with the complex. We concluded that unlike GCPs 2-6, which are important for $\gamma$ TuRC assembly (Verollet et al., 2006; Xiong and Oakley, 2009), GCP8 has no structural role in the $\gamma$ TuRC.

\section{GCP8 Depletion Impairs Recruitment of $\gamma \mathrm{TuRC}$ Components to Interphase Centrosomes}

$\gamma \mathrm{TuRC}$ recruitment and microtubule nucleation activity are essential for the microtubule organizing function of centrosomes. To investigate whether GCP8 depletion affects centrosomal $\gamma \mathrm{TuRC}$ we costained control and GCP8-depleted cells with antibodies against GCP8 and various centrosome components. At interphase centrosomes with strongly reduced levels of GCP8 we observed a reduction of both GCP-WD and $\gamma$-tubulin ( $\sim 30 \%$ reduction, respectively) (Figure 5A). Interestingly, GCP8 depletion caused an even stronger reduction in the amount of centrosomal CDK5RAP2 ( $\sim 45 \%$ reduction). Importantly, the levels of pericentrin, a component of the PCM, were only slightly reduced $(\sim 15 \%$ reduction) and staining with the centriole marker centrin revealed the presence of normal numbers of centrioles (Figure 5A and Supplemental Figure S4), indicating that GCP8 depletion did not disrupt the general centrosome structure. This conclusion is further supported by FACS analysis, which failed to detect a significant increase in the G1/S population of cells, suggesting that GCP8 depletion does not induce a specific G1/S arrest, as previously reported for RNAi treatments that compromise centrosome integrity (Supplemental Figure S3). Interestingly, depletion of GCP8 did not impair the localization of $\gamma$ TuRC components to centrosomes in mitosis (Figure 5B), suggesting a specific effect on interphase centrosomes. In mitotic cells depleted of GCP8 localization of $\gamma$-tubulin to spindle microtubules was also unaffected (Figure 5B).

\section{GCP8 Depletion Inhibits Microtubule Nucleation at Interphase Centrosomes}

We next tested whether centrosomes in GCP8-depleted cells were able to nucleate microtubules. We performed a cellbased microtubule regrowth assay, in which cells were coldtreated to completely depolymerize microtubules and then incubated at $37^{\circ} \mathrm{C}$ to allow microtubule nucleation and regrowth. After $10 \mathrm{~s}$ of regrowth, small microtubule asters had formed at centrosomes in both control and GCP8-depleted cells (Figure 6A). However, centrosomal microtubule asters in GCP8-depleted cells were slightly smaller than in control cells and quantification of the microtubule signal surrounding the centrosomes indicated a $\sim 30 \%$ reduction (Figure 6A). Again, the effect was specific to interphase centrosomes as we could not detect differences in nucleation activity between control and GCP8-depleted centrosomes in mitosis (Figure 6B). In summary, GCP8 depletion impairs the nucleation activity of centrosomes in interphase by interfering with the centrosomal recruitment of $\gamma \mathrm{TuRC}$ components specifically during this phase of the cell cycle.

\section{DISCUSSION}

We have developed a tandem affinity-based method that allows the efficient purification of $\gamma \mathrm{TuRC}$ from human cells while favoring protein-protein interactions. Our protocol includes a polyethylene glycol precipitation step to selectively enrich $\gamma$ TuRC (Murphy et al., 2001). Even though we cannot rule out that in addition to $\gamma \mathrm{TuRC}$ other $\gamma$-tubulin containing protein complexes were copurified, the most abundant polypeptides in our preparations were almost exclusively $\gamma$ TuRC subunits, and their relative amounts were very similar to those of immunoaffinity-purified $\gamma$ TuRCs in previous studies (Zheng et al., 1995; Moritz et al., 1998; Murphy et al., 1998; Fava et al., 1999; Oegema et al., 1999; Murphy et al., 2001), suggesting that our method is well 
A

Homo sapiens GCP8A

Homo sapiens GCP8B Mus musculus

Danio rerio

Branchiostoma floridae

Strongylocentrotus purpuratus

Homo sapiens GCP8A

Mus musculus

Xenopus tropicalis

Danio rerio

Stror
Xenopus tropicalis

Nematostella vectensis

Homo sapiens GCP8B

Branchiostoma floridae
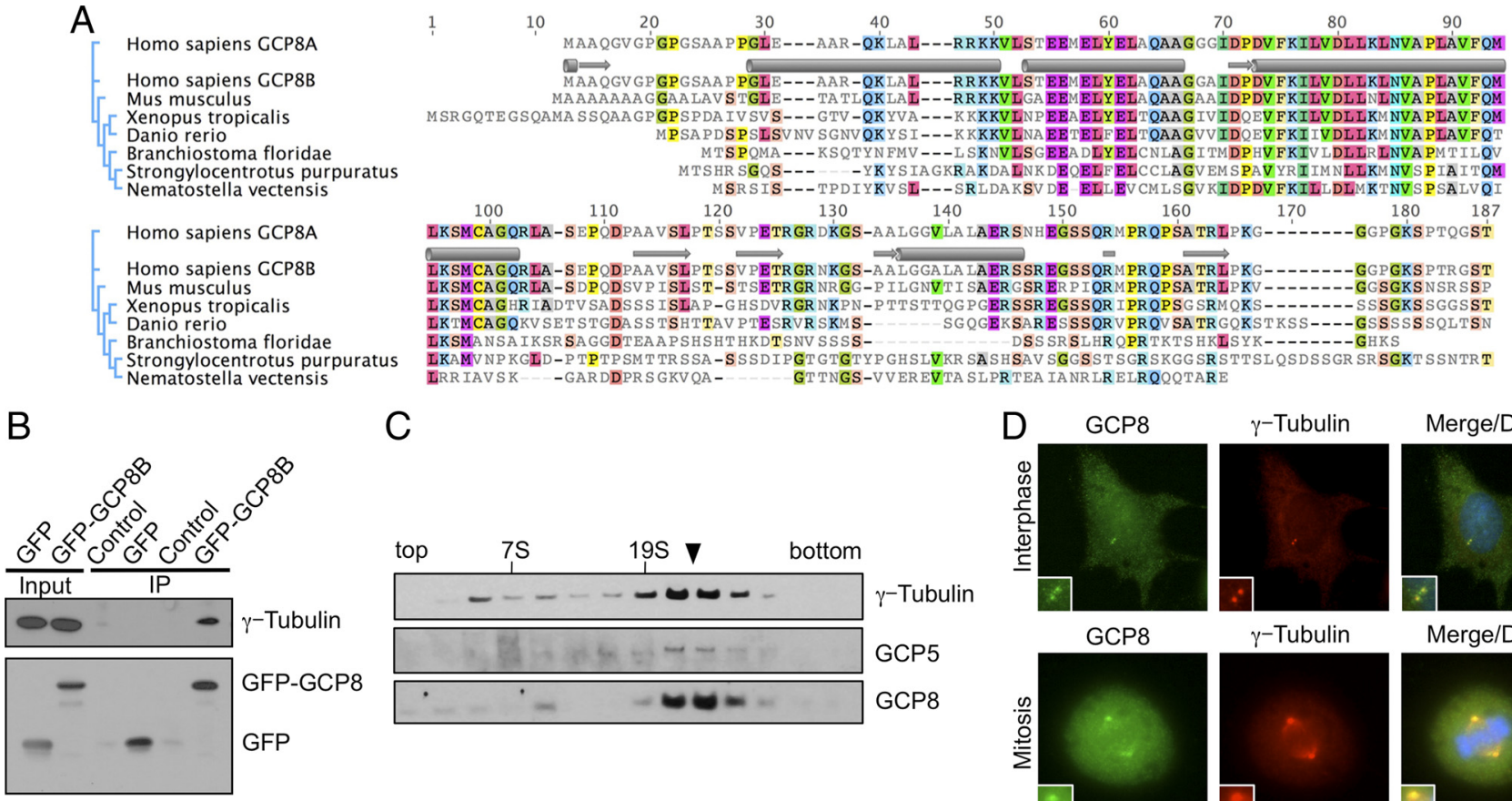

C

D

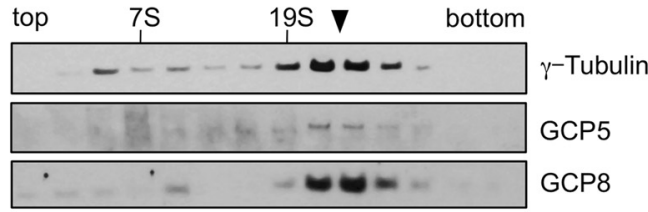

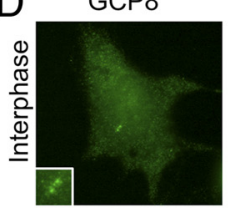

GCP8

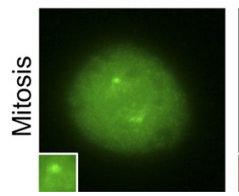

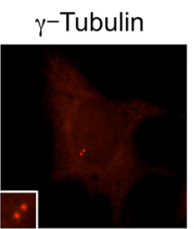

$\gamma$-Tubulin

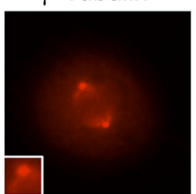

Merge/DNA

Figure 2. GCP8 has properties of a $\gamma \mathrm{TuRC}$ subunit. (A) Amino acid sequence alignment of human GCP8A/B and putative orthologues from various species as indicated. Conserved amino acids (identical in at least $50 \%$ of the aligned sequences) are shaded in colors. The relationship between sequences is indicated by a phylogenetic tree on the left. Predicted secondary structures are indicated for human GCP8B above its sequence. Helical regions are indicated by a tube symbol, $\beta$-sheets by a block arrow symbol. (B) GFP and GFP-GCP8B, respectively, were transiently expressed in Hela cells and immunoprecipitated with anti-GFP antibody. After Western blotting the immunoprecipitates were probed with antibodies against the indicated proteins. (C) Extract of U2OS cells was fractionated by sucrose gradient centrifugation. Fractions were analyzed by Western blotting with antibodies against the indicated proteins. The arrowhead marks the $\gamma$ TuRC peak fraction. Aldolase $(158 \mathrm{kDa}, 7 \mathrm{~S})$ and thyroglobulin $(669 \mathrm{kDa}, 19 \mathrm{~S})$ were used as molecular weight standards. (D) U2OS cells were fixed and stained with antibodies against GCP8 and $\gamma$-tubulin as indicated. DAPI was used to stain DNA. Insets show magnified centrosome areas. Scale bar, $10 \mu \mathrm{m}$.

suited to purify $\gamma \mathrm{TuRC}$. Here we have used purified $\gamma \mathrm{TuRC}$ for a comprehensive analysis of their composition and interactions at distinct cell cycle stages by mass spectrometry.

\section{Core Components of the Human $\gamma \mathrm{TuRC}$}

Based on our analysis we can redefine the set of human $\gamma \mathrm{TuRC}$ core subunits, which account for the most abundant polypeptides present in purified $\gamma$ TuRC and are found in both interphase and mitotic complexes. This set of subunits comprises $\gamma$-tubulin, GCPs 2-6, GCP-WD, and the novel previously uncharacterized protein GCP8. In a previous study published during the preparation of this manuscript, GCP8 was termed MOZART2 (Mitotic spindle organizing protein associated with a ring of $\gamma$-tubulin) (Hutchins et al.,
A

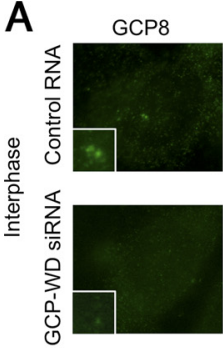

$\gamma$-Tubulin
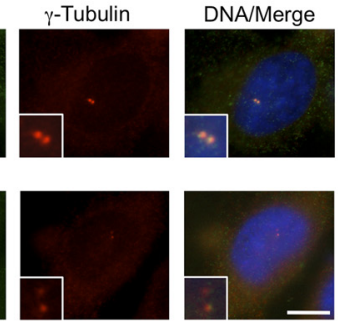

B
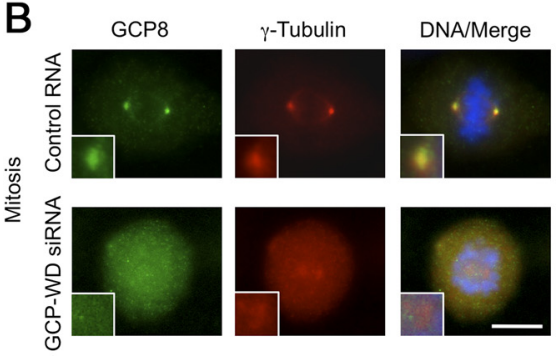
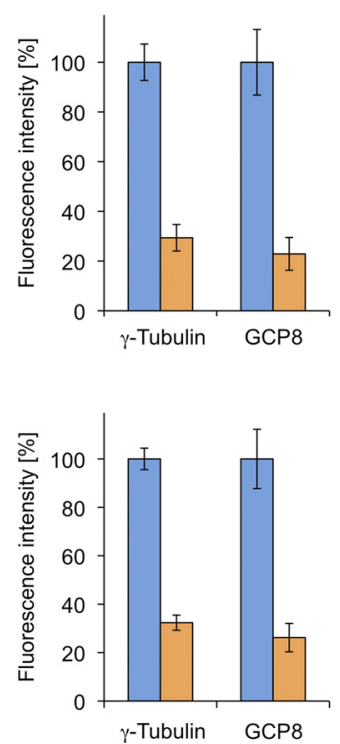

$\square$ Control RNA

$\square$ GCP-WD SIRNA

$\square$ Control RNA

$\square$ GCP-WD siRNA

Figure 3. Centrosome targeting of GCP8 requires GCP-WD. (A) Interphase U2OS cells transfected with either control RNA or siRNA against GCP-WD were stained with $\gamma$-tubulinand GCP8-specific antibodies. DNA was stained with DAPI. The insets show magnifications of centrosomal areas. The fluorescence intensities of centrosomal $\gamma$-tubulin and GCP8 staining were quantified, and the centrosomal signal measured in control cells was set to $100 \%$. Mean values are plotted as percentages of intensities in control cells ( $n>20$, error bars: SEM). (B) Mitotic U2OS cells analyzed as in A. Scale bars, $10 \mu \mathrm{m}$. 
Figure 4. Depletion of GCP8 does not affect assembly or stability of the $\gamma \mathrm{TuRC}$. (A) U2OS cells were transfected with control RNA or siRNA targeting GCP8. After $72 \mathrm{~h}$ whole cell lysates were analyzed by Western blotting with antibodies against the indicated proteins. (B) Equal numbers of control-transfected cells and cells transfected with GCP8 siRNA were seeded on culture dishes and grown for $72 \mathrm{~h}$. After harvesting the cell numbers were determined by counting. Mean values from four independent transfections were plotted. Error bars: SEM. (C) U2OS cells were transfected with control RNA or with siRNA targeting GCP8. After $72 \mathrm{~h}$ cells were lysed and extracts fractionated by sucrose gradient centrifugation. Fractions were analyzed by Western blotting and probed with antibodies against the indicated proteins. The arrowhead marks the $\gamma$ TuRC peak fraction. Peak fractions of aldolase $(158 \mathrm{kDa}, 7 \mathrm{~S})$ and thyroglobulin $(669 \mathrm{kDa}, 19 \mathrm{~S})$ as molecular weight standards are indicated.
2010). In agreement with this study our results suggest that GCP8/MOZART2 does not play an important role in spindle assembly. In addition, we show that GCP8 has properties of a $\gamma \mathrm{TuRC}$ core subunit. We would like to propose the uniform "GCP" nomenclature for all core components of the human $\gamma \mathrm{TuRC}$, as identified in our study (Murphy et al., 1998). Accordingly, we suggest the term GCP8 for MOZART2 (counting GCP-WD/NEDD1 as GCP7). Even though GCP-WD and GCP8 are not sequence-related to GCPs 2-6, this simplified nomenclature appreciates that all these proteins qualify as true $\gamma \mathrm{TuRC}$ subunits by standard assays.
A

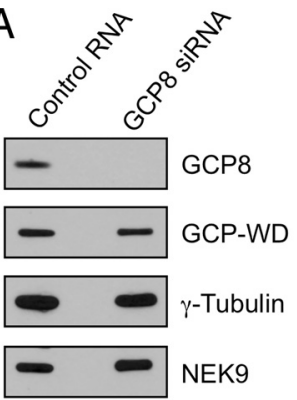

C
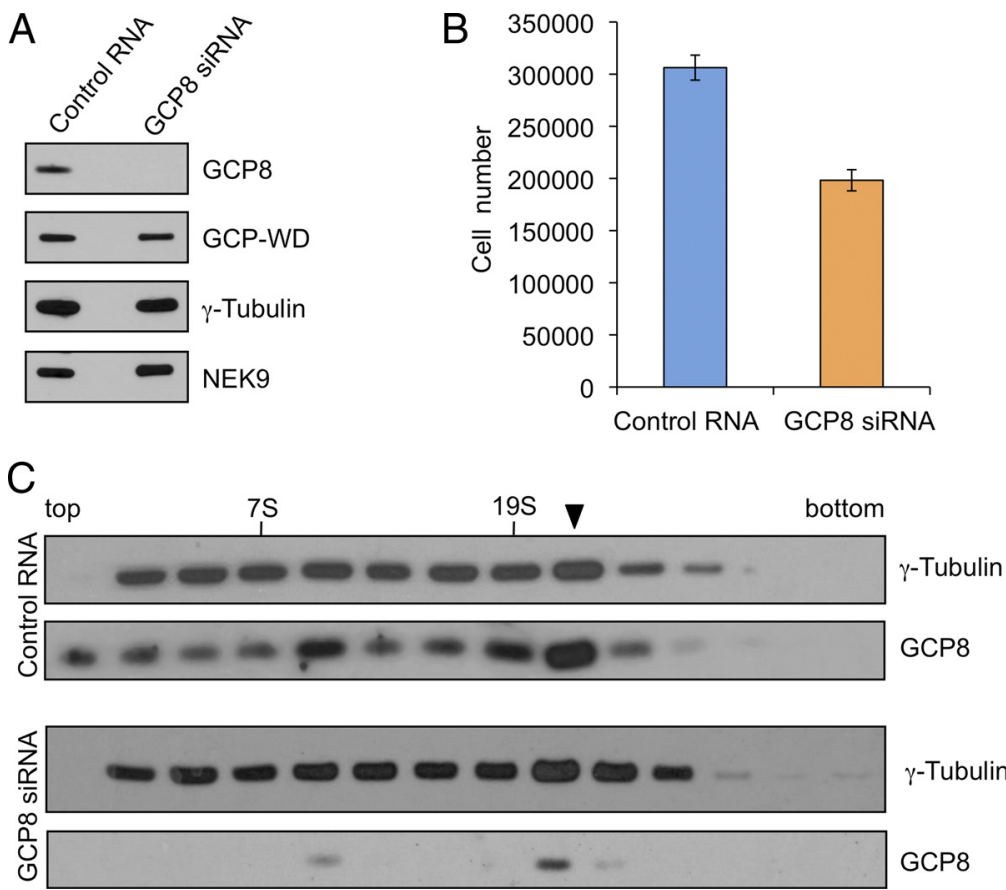

ur study shows that MOZART1, another mitotic interactor of $\gamma$ TuRCs (Hutchins et al., 2010), is also present in interphase $\gamma$ TuRCs. However, due to its small size $(8.5 \mathrm{kDa})$ we did not detect this protein in our initial analysis by PAGE and Coomassie staining and cannot assess its abundance in the $\gamma$ TuRC. Future studies will show whether MOZART1 qualifies as an additional core subunit of the $\gamma \mathrm{TuRC}$.

\section{$\gamma$ TuRC Interactors}

Apart from the $\gamma \mathrm{TuRC}$ core subunits we have also identified additional proteins present in purified $\gamma \mathrm{TuRCs}$. As these proteins are less abundant than the core subunits, their
Figure 5. Depletion of GCP8 interferes with phase centrosomes. U2OS cells transfected with either control RNA or siRNA against GCP8 were stained with antibodies against various centrosome proteins. As an example, interphase (A) and mitotic (B) cells stained with GCP8 and $\gamma$-tubulin antibodies are shown. DNA was visualized with DAPI. Scale bars, $10 \mu \mathrm{m}$. The fluorescence intensities of centrosomal signals were quantified for each of the detected proteins in interphase (A) and mitotic (B) cells, respectively. Mean values are plotted as percentages of intensities in control cells, which were set to $100 \%$ ( $n>20$, error bars: SEM). the recruitment of $\gamma \mathrm{TuRC}$ components to inter-
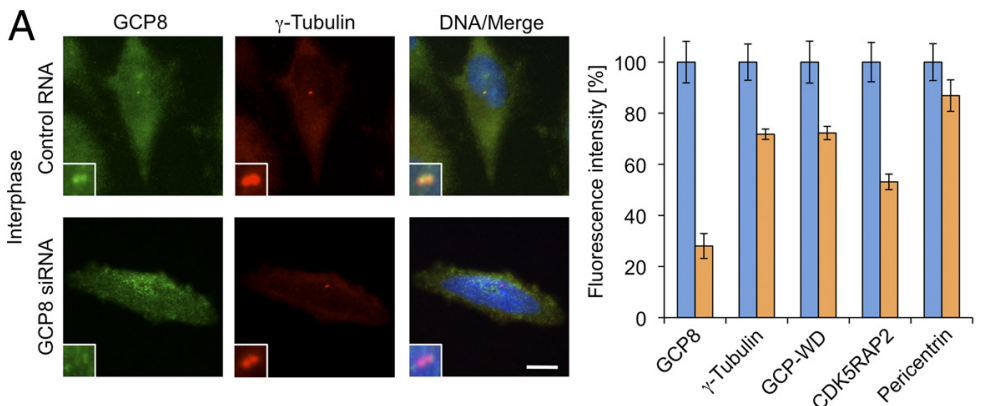

$\square$ Control RNA 口GCP8 SIRNA
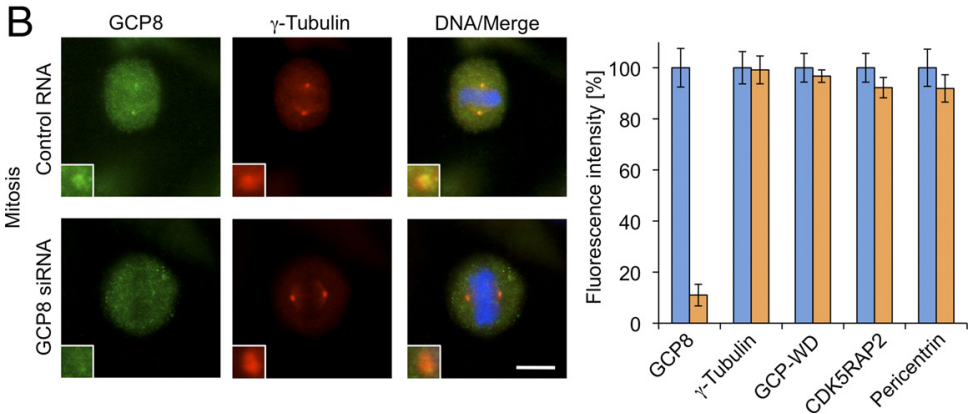

$\square$ Control RNA 口GCP8 SiRNA 
A

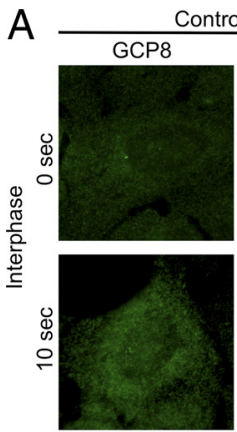
Control RNA

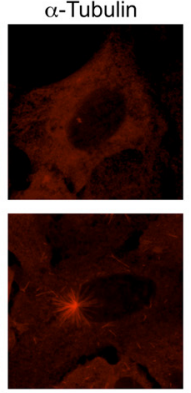

B

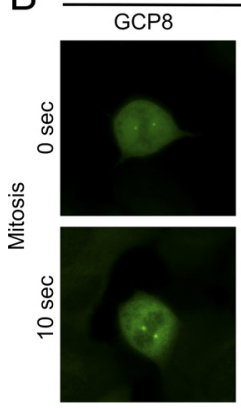

ontrol RNA

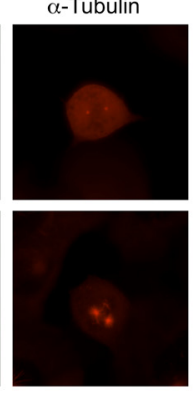

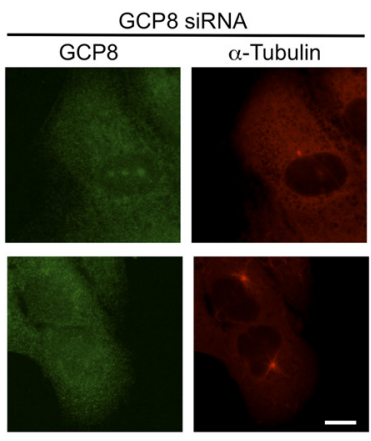

GCP8 SiRNA

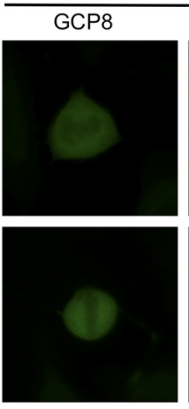

$\alpha$-Tubulin

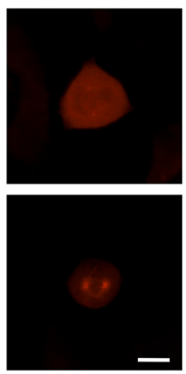

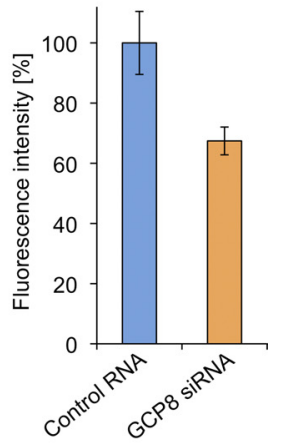

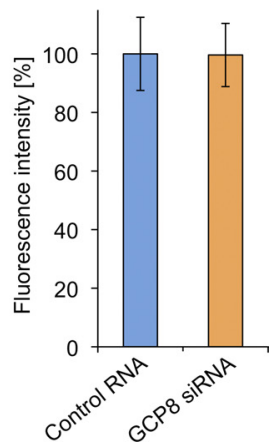

Figure 6. Depletion of GCP8 interferes with interphase centrosomal microtubule nucleation. (A) Nonsynchronized U2OS cells were transfected either with control RNA or GCP8 siRNA and were subjected to a microtubule regrowth assay. Microtubules were depolymerized on ice (time point 0 ) and after warming microtubules were allowed to regrow (time point $10 \mathrm{~s}$ ) before fixation and staining with antibodies against GCP8 and $\alpha$-tubulin. Scale bar, $10 \mu \mathrm{m}$. The intensities of the microtubule asters that had formed around centrosomes in interphase after $10 \mathrm{~s}$ of regrowth were quantified and mean values were plotted as percentages of intensities in control cells $(n>20$, error bars: SEM). (B) U2OS cells transfected either with control RNA or GCP8 siRNA were arrested in mitosis with nocodazole. Cells were subjected to a microtubule regrowth assay and analyzed as in A. Scale bar, $10 \mu \mathrm{m}$. The intensities of the microtubule asters that had formed around centrosomes in mitotic cells after $10 \mathrm{~s}$ of regrowth were quantified, and mean values were plotted as percentages of intensities in control cells ( $n>20$, error bars: SEM). interaction with the $\gamma$ TuRC might be of lower affinity or be subject to regulation. Examples of such interactors present in both interphase and mitotic $\gamma \mathrm{TuRC}$ are the nucleosidediphosphate kinase NME7, subunits of the CCT chaperonin, the centrosomal protein CDK5RAP2, and the AAA+ ATPase RUVBL1. NME7 belongs to a family of nucleoside diphosphate kinases, which play an important role in the generation of nucleoside triphosphates such as ATP and GTP. Interestingly, a Chlamydomonas protein with homology to NME7 was identified as a component of basal bodies and of the flagellum (Keller et al., 2005; Pazour et al., 2005), and the characterization of NME7 knockout mice suggested a function in ciliary motility (Vogel et al., 2010). Whether human NME7 also has a function in ciliary motility and what role the interaction with the $\gamma \mathrm{TuRC}$ might play is currently unclear. The CCT chaperonin complex binds to unfolded $\gamma$-tubulin and promotes its proper folding (Melki et al., 1993). As the $\gamma$ TuRC purification via tagged $\gamma$-tubulin might result in the copurification of some partially folded $\gamma$-tubulin, the presence of chaperonin subunits in our $\gamma \mathrm{TuRC}$ preparations is not surprising. Our $\gamma \mathrm{TuRC}$ preparations also contained CDK5RAP2, a structural component of the PCM participating in $\gamma \mathrm{TuRC}$ recruitment (Fong et al., 2008; Haren et al., 2009), whereas pericentrin, for which a similar function has been described, was not detected (Zimmerman et al., 2004; Haren et al., 2009). One interpretation would be that the interaction between pericentrin and the $\gamma \mathrm{TuRC}$ is restricted to the centrosome (Dictenberg et al., 1998), whereas CDK5RAP2 might also associate with noncentrosomal $\gamma \mathrm{TuRC}$. In this context it is interesting to note that both CDK5RAP2 and $\gamma \mathrm{TuRC}$ have recently been implicated in the centrosome-independent regulation of microtubule plus end dynamics (Fong et al., 2009; Bouissou et al., 2009). RUVBL1 and RUVBL2 have both been described as $\gamma$ TuRC interactors, and RUVBL1 was shown to have a role in mitotic spindle assembly (Ducat et al., 2008). However, we find that both proteins are also associated with interphase $\gamma \mathrm{TuRC}$ suggesting that these proteins might also play a role in nonmitotic functions of the $\gamma \mathrm{TuRC}$. Addressing this question will be a major challenge as RUVBL1 and RUVBL2 have been implicated in multiple essential cellular processes such as chromatin remodeling, DNA repair, and transcription. Interestingly, CDK5RAP2, RUVBL1 and RUVBL2 were not identified as $\gamma \mathrm{TuRC}$ interactors in the large-scale analysis of mitotic protein complexes (Hutchins et al., 2010). The different tagging strategy and method used for the purification of the $\gamma \mathrm{TuRCs}$ might explain these differences.

\section{Mitosis-Specific $\gamma$ TuRC Interactors}

Another category of transient interactors are proteins that interact with $\gamma \mathrm{TuRC}$ in a cell cycle-dependent manner. In preparations of mitotic $\gamma \mathrm{TuRC}$ we identified seven of the eight subunits of the human augmin complex, most of which could not be detected in $\gamma$ TuRCs obtained from interphase cells. A mitosis-specific interaction with $\gamma \mathrm{TuRC}$ components has been demonstrated previously for the augmin subunit HAUS6 (Zhu et al., 2008). Our results indicate that this interaction most likely involves the entire augmin complex and highlights the importance of this interaction for the function of the $\gamma \mathrm{TuRC}$ in noncentrosomal microtubule formation during mitotic spindle assembly (Luders et al., 2006; Luders and Stearns, 2007; Goshima et al., 2008). In addition to augmin we also identified other proteins preferentially associated with mitotic $\gamma$ TuRCs including the mitotic kinase Plk1, for which an interaction with the $\gamma$ TuRC subunit GCP-WD has been demonstrated previously (Haren et al., 2009; Zhu et al., 2009). It will be interesting to test whether Plk1 also binds and potentially regulates other components of the $\gamma$ TuRC.

\section{The Novel Subunit GCP8}

GCP8, the novel $\gamma$ TuRC subunit described in this study, is not sequence-related to GCPs 2-6 and does not seem to have a structural role in the $\gamma \mathrm{TuRC}$. GCP8 shares this property with the recently described GCP-WD subunit, suggesting that both proteins might have regulatory functions. In agree- 
ment with this interpretation GCP-WD was recently shown to function as a centrosomal attachment factor for the $\gamma \mathrm{TuRC}$ and to be regulated by mitotic phosphorylation (Luders et al., 2006; Haren et al., 2006). Our results indicate that GCP8 depletion reduces centrosomal microtubule nucleation by interfering with the recruitment of both GCP-WD and $\gamma$-tubulin specifically during interphase. This interphase-specific role of GCP8, which is unique among the characterized $\gamma$ TuRC subunits, might explain why GCP8depleted cells did not display obvious mitotic defects. We also did not observe centriole duplication defects after GCP8 depletion. It is possible that the remaining nucleation activity at GCP8 depleted centrosomes is sufficient to support centriole duplication or that GCP8 depletion selectively depletes a centrosomal fraction of $\gamma$-tubulin that might not be involved in centriole duplication. Indeed, distinct subpopulations of centrosomal $\gamma$-tubulin have been described (Khodjakov and Rieder, 1999).

What are the consequences of the defects in centrosomal $\gamma$-tubulin recruitment and microtubule nucleation in GCP8depleted cells? It is tempting to speculate that changes in the organization of the interphase microtubule network might play a role in the reduced proliferation rate of GCP8-depleted cells. However, a recent study has demonstrated that cells with a compromised or even completely depolymerized microtubule network can progress through interphase with near-normal kinetics (Uetake and Sluder, 2007). Microtubules were present in GCP8-depleted cells, but we cannot exclude more subtle defects such as changes in microtubule dynamics. GCP8 might also play a role in noncentrosomal $\gamma$ TuRC functions. For example, the $\gamma \mathrm{TuRC}$ was recently shown to localize along interphase microtubules and to regulate microtubule plus end dynamics in Drosophila S2 cells (Bouissou et al., 2009).

We could not identify GCP8-related sequences in yeast, nematodes, flies, or plants, however we cannot rule out the existence of functional homologues with a low degree of conservation at the primary amino acid sequence level. Alternatively, GCP8 might be less important for cells that do not actively nucleate microtubules at centrosomes in interphase. In Drosophila, for example, interphase centrosomes do not recruit detectable amounts of $\gamma$-tubulin and do not function as MTOCs (Rogers et al., 2008).

In this study we have identified and extended the set of $\gamma$ TuRC core subunits and provided a comprehensive list of $\gamma \mathrm{TuRC}$ interactors. Together with previous studies our work lays grounds for detailed future analyses of the roles of distinct $\gamma \mathrm{TuRC}$ interactors in microtubule organization in both interphase and mitosis.

\section{ACKNOWLEDGMENTS}

We thank Aaron Groen and Ryoma Ohi for the kind gift of the anti-centrin antibody. This work was supported by the Plan Nacional of I+D+I grants BFU-2007-62087 (to C.C.), BFU2008-03441 (to J.R.) and BFU2009-08522 (to J.L.) (Ministerio de Ciencia e Innovación, Spain). In addition, J.L. acknowledges support from a Marie Curie International Reintegration Grant (FP7-PEOPLE2007-4-3-IRG, project \#224835) and from IRB intramural funds. J.R. is supported by the Ramón y Cajal Program (MICINN, Spain), N.T. is the recipient of an IRB Barcelona Interprogram Postdoctoral Fellowship, and M.T.B. is a recipient of a FPU fellowship (MICINN, Spain).

\section{REFERENCES}

Bouissou, A., Verollet, C., Sousa, A., Sampaio, P., Wright, M., Sunkel, C. E., Merdes, A., and Raynaud-Messina, B. (2009). \{gamma\}-Tubulin ring complexes regulate microtubule plus end dynamics. J. Cell Biol. 187, 327-334.

Dictenberg, J. B., Zimmerman, W., Sparks, C. A., Young, A., Vidair, C., Zheng, Y., Carrington, W., Fay, F. S., and Doxsey, S. J. (1998). Pericentrin and gamma-tubulin form a protein complex and are organized into a novel lattice at the centrosome. J. Cell Biol. 141, 163-174.

Ducat, D., Kawaguchi, S., Liu, H., Yates, J. R., 3rd, and Zheng, Y. (2008) Regulation of microtubule assembly and organization in mitosis by the AAA+ ATPase Pontin. Mol. Biol. Cell 19, 3097-3110.

Elias, J. E., and Gygi, S. P. (2007). Target-decoy search strategy for increased confidence in large-scale protein identifications by mass spectrometry. Nat. Methods 4, 207-214.

Fava, F., Raynaud-Messina, B., Leung-Tack, J., Mazzolini, L., Li, M Guillemot, J. C., Cachot, D., Tollon, Y., Ferrara, P., and Wright, M. (1999). Human 76p: A new member of the gamma-tubulin-associated protein family. J. Cell Biol. 147, 857-868.

Fong, K. W., Choi, Y. K., Rattner, J. B., and Qi, R. Z. (2008). CDK5RAP2 is a pericentriolar protein that functions in centrosomal attachment of the fgamma\}-tubulin ring complex. Mol. Biol. Cell 19, 115-125.

Fong, K. W., Hau, S. Y., Kho, Y. S., Jia, Y., He, L., and Qi, R. Z. (2009). Interaction of CDK5RAP2 with EB1 to track growing microtubule tips and to regulate microtubule dynamics. Mol. Biol. Cell 20, 3660-3670.

Fujita, A., Vardy, L., Garcia, M. A., and Toda, T. (2002). A fourth component of the fission yeast gamma-tubulin complex, Alp16, is required for cytoplasmic microtubule integrity and becomes indispensable when gamma-tubulin function is compromised. Mol. Biol. Cell 13, 2360-2373.

Goshima, G., Mayer, M., Zhang, N., Stuurman, N., and Vale, R. D. (2008). Augmin: a protein complex required for centrosome-independent microtubule generation within the spindle. J. Cell Biol. 181, 421-429.

Groen, A. C., Cameron, L. A., Coughlin, M., Miyamoto, D. T., Mitchison, T. J., and Ohi, R. (2004). XRHAMM functions in ran-dependent microtubule nucleation and pole formation during anastral spindle assembly. Curr. Biol. 14, 1801-1811.

Haren, L., Remy, M. H., Bazin, I., Callebaut, I., Wright, M., and Merdes, A. (2006). NEDD1-dependent recruitment of the gamma-tubulin ring complex to the centrosome is necessary for centriole duplication and spindle assembly. J. Cell Biol. 172, 505-515

Haren, L., Stearns, T., and Luders, J. (2009). Plk1-dependent recruitment of gamma-tubulin complexes to mitotic centrosomes involves multiple PCM components. PLoS One 4, e5976

Hutchins, J. R., et al. (2010). Systematic analysis of human protein complexes identifies chromosome segregation proteins. Science 328, 593-599.

Keller, L. C., Romijn, E. P., Zamora, I., Yates, J. R., 3rd, and Marshall, W. F. (2005). Proteomic analysis of isolated chlamydomonas centrioles reveals orthologs of ciliary-disease genes. Curr. Biol. 15, 1090-1098.

Khodjakov, A., and Rieder, C. L. (1999). The sudden recruitment of gammatubulin to the centrosome at the onset of mitosis and its dynamic exchange throughout the cell cycle, do not require microtubules. J. Cell Biol. 146, $585-596$

Kollman, J. M., Zelter, A., Muller, E. G., Fox, B., Rice, L. M., Davis, T. N., and Agard, D. A. (2008). The Structure of the \{gamma\}-tubulin small complex: implications of its architecture and flexibility for microtubule nucleation. Mol. Biol. Cell 19, 207-215.

Lawo, S., et al. (2009). HAUS, the 8-subunit human augmin complex, regulates centrosome and spindle integrity. Curr. Biol. 19, 816-826.

Luders, J., Patel, U. K., and Stearns, T. (2006). GCP-WD is a gamma-tubulin targeting factor required for centrosomal and chromatin-mediated microtubule nucleation. Nat. Cell Biol. 8, 137-147.

Luders, J., and Stearns, T. (2007). Microtubule-organizing centres: a re-evaluation. Nat. Rev. Mol. Cell Biol. 8, 161-167.

Ma, W., Baumann, C., and Viveiros, M. M. (2010). NEDD1 is crucial for meiotic spindle stability and accurate chromosome segregation in mammalian oocytes. Dev. Biol. 339, 439-450.

Melki, R., Vainberg, I. E., Chow, R. L., and Cowan, N. J. (1993). Chaperoninmediated folding of vertebrate actin-related protein and gamma-tubulin. J. Cell Biol. 122, 1301-1310.

Moritz, M., Braunfeld, M. B., Guenebaut, V., Heuser, J., and Agard, D. A. (2000). Structure of the gamma-tubulin ring complex: a template for microtubule nucleation. Nat. Cell Biol. 2, 365-370.

Moritz, M., Zheng, Y., Alberts, B. M., and Oegema, K. (1998). Recruitment of the gamma-tubulin ring complex to Drosophila salt-stripped centrosome scaffolds. J. Cell Biol. 142, 775-786.

Murphy, S. M., Preble, A. M., Patel, U. K., O'Connell, K. L., Dias, D. P., Moritz M., Agard, D., Stults, J. T., and Stearns, T. (2001). GCP5 and GCP 6, two new members of the human gamma-tubulin complex. Mol. Biol. Cell 12, 33403352. 


\section{N. Teixidó-Travesa et al.}

Murphy, S. M., Urbani, L., and Stearns, T. (1998). The mammalian gammatubulin complex contains homologues of the yeast spindle pole body components spc97p and spc98p. J. Cell Biol. 141, 663-674.

Oegema, K., Wiese, C., Martin, O. C., Milligan, R. A., Iwamatsu, A., Mitchison, T. J., and Zheng, Y. (1999). Characterization of two related Drosophila gamma-tubulin complexes that differ in their ability to nucleate microtubules. J. Cell Biol. 144, 721-733.

Pazour, G. J., Agrin, N., Leszyk, J., and Witman, G. B. (2005). Proteomic analysis of a eukaryotic cilium. J. Cell Biol. 170, 103-113.

Rigaut, G., Shevchenko, A., Rutz, B., Wilm, M., Mann, M., and Seraphin, B. (1999). A generic protein purification method for protein complex characterization and proteome exploration. Nat. Biotechnol. 17, 1030-1032.

Rogers, G. C., Rusan, N. M., Peifer, M., and Rogers, S. L. (2008). A multicomponent assembly pathway contributes to the formation of acentrosomal microtubule arrays in interphase Drosophila cells. Mol. Biol. Cell 19, 3163-3178.

Roig, J., Mikhailov, A., Belham, C., and Avruch, J. (2002). Nercc1, a mammalian NIMA-family kinase, binds the Ran GTPase and regulates mitotic progression. Genes Dev. 16, 1640-1658.

Uehara, R., Nozawa, R. S., Tomioka, A., Petry, S., Vale, R. D., Obuse, C., and Goshima, G. (2009). The augmin complex plays a critical role in spindle microtubule generation for mitotic progression and cytokinesis in human cells. Proc. Natl. Acad. Sci. USA. 106, 6998-7003.

Uetake, Y., and Sluder, G. (2007). Cell-cycle progression without an intact microtuble cytoskeleton. Curr. Biol. 17, 2081-2086.

Venkatram, S., Tasto, J. J., Feoktistova, A., Jennings, J. L., Link, A. J., and Gould, K. L. (2004). Identification and characterization of two novel proteins affecting fission yeast gamma-tubulin complex function. Mol. Biol. Cell 15 2287-2301.

Verollet, C., Colombie, N., Daubon, T., Bourbon, H.M., Wright, M., and Raynaud-Messina, B. (2006). Drosophila melanogaster gamma-TuRC is dispensable for targeting gamma-tubulin to the centrosome and microtubule nucleation. J. Cell Biol. 172, 517-528.

Vogel, P., Read, R., Hansen, G. M., Freay, L. C., Zambrowicz, B. P., and Sands, A. T. (2010). Situs inversus in Dpcd/Poll-/-, Nme7-/-, and Pkd111-/mice. Vet. Pathol. 47, 120-131.

Xiong, Y., and Oakley, B. R. (2009). In vivo analysis of the functions of gamma-tubulin-complex proteins. J. Cell Sci. 122, 4218-4227.

Zeng, C. J., Lee, Y. R., and Liu, B. (2009). The WD40 repeat protein NEDD1 functions in microtubule organization during cell division in Arabidopsis thaliana. Plant Cell 21, 1129-1140.

Zheng, Y., Wong, M. L., Alberts, B., and Mitchison, T. (1995). Nucleation of microtubule assembly by a gamma-tubulin-containing ring complex. Nature $378,578-583$

Zhu, H., Coppinger, J. A., Jang, C. Y., Yates, J. R., 3rd, and Fang, G. (2008) FAM29A promotes microtubule amplification via recruitment of the NEDD1gamma-tubulin complex to the mitotic spindle. J. Cell Biol. 183, 835-848.

Zhu, H., Fang, K., and Fang, G. (2009). FAM29A, a target of Plk1 regulation, controls the partitioning of NEDD1 between the mitotic spindle and the centrosomes. J. Cell Sci. 122, 2750-2759.

Zimmerman, W. C., Sillibourne, J., Rosa, J., and Doxsey, S. J. (2004). Mitosisspecific anchoring of gamma tubulin complexes by pericentrin controls spindle organization and mitotic entry. Mol. Biol. Cell 15, 3642-3657. 